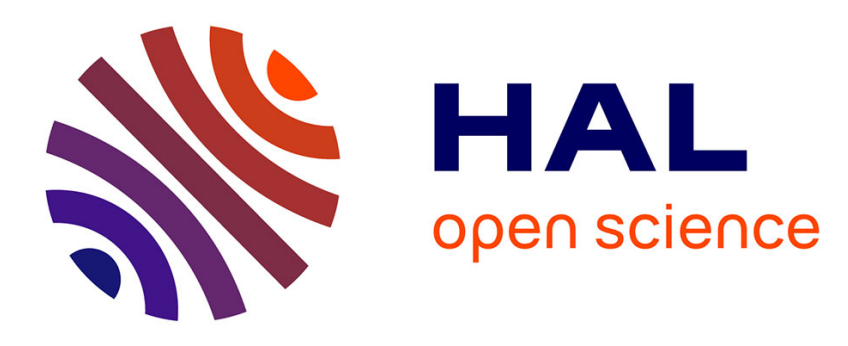

\title{
Tolerance synthesis using bond graph inversion and fuzzy logic
}

van Hoa Nguyen, Damien Eberard, Wilfrid Marquis-Favre, Laurent

Krähenbühl

\section{- To cite this version:}

van Hoa Nguyen, Damien Eberard, Wilfrid Marquis-Favre, Laurent Krähenbühl. Tolerance synthesis using bond graph inversion and fuzzy logic. ICM International Conference on Mechatronics, Feb 2013, Vicenza, Italy. pp.442-447, 10.1109/ICMECH.2013.6518577 . hal-00786671

\section{HAL Id: hal-00786671 https://hal.science/hal-00786671}

Submitted on 24 Apr 2019

HAL is a multi-disciplinary open access archive for the deposit and dissemination of scientific research documents, whether they are published or not. The documents may come from teaching and research institutions in France or abroad, or from public or private research centers.
L'archive ouverte pluridisciplinaire HAL, est destinée au dépôt et à la diffusion de documents scientifiques de niveau recherche, publiés ou non, émanant des établissements d'enseignement et de recherche français ou étrangers, des laboratoires publics ou privés. 


\title{
Tolerance synthesis using bond graph inversion and fuzzy logic
}

\author{
Van Hoa NGUYEN*, Damien EBERARD*, Wilfrid MARQUIS-FAVRE* and Laurent KRAHENBUHL ${ }^{\dagger}$ \\ *Université de Lyon, INSA de Lyon, Laboratoire Ampère (CNRS UMR5005), \\ Address: 20 Avenue Albert Einstein 69621 Villeurbanne cedex - France, \\ Email: surname.name@insa-lyon.fr \\ †Université de Lyon, École Centrale de Lyon, Laboratoire Ampère (CNRS UMR5005) \\ Address: 36 avenue Guy de Collongue - 69134 Ecully Cedex - France, \\ Email: laurent.krahenbuhl@ec-lyon.fr
}

\begin{abstract}
In the context of mechatronic systems design, this paper addresses a parameter tolerance synthesis with respect to specifications including output epistemic uncertainties. The methodology proposed here concerns uncertainties modelled with fuzzy logic. The procedure relies on output uncertainties propagation through an inverse model. Design parameter tolerance is then synthesized. The results are validated injecting designed parameters in the direct model. The methodology is illustrated on a linear model with specifications including combined uncertainties.
\end{abstract}

\section{INTRODUCTION}

In the last few decades, mechatronic and its applications have dramatically changed the world. As a consequence, the problem of mechatronic system design has drawn more and more attention.

The sizing problem takes an important place in mechatronic system design. In the sizing process, possible technical solutions are determined in order to satisfy a set of requirements. A certain number of sizing methodologies have been proposed in literature. Roughly speaking, they are classified into two categories: direct methods (trial - error - correction) and inverse methods (inputs are deduced from desired outputs). The direct methods are very popular and are applied at large scale in industry. However, they are expensive in computational time. The inverse method provides designers with a less computational solution for sizing problem. However it may be difficult to apply, since the invertibility condition of the model is required.

It is worth noting that the sizing problem is deterministic. In reality, there is always a difference between the observed real system's performance and the mathematical model's performance. There are many reasons for that difference: variability during manufacturing process, sensors sensibility, modelling assumptions, etc... The rising question is: which tolerance can we apply on the design parameters of the system to keep satisfying the requirements?

In the frame of our work, we are interested in parameter tolerance synthesis with respect to specifications including output epistemic uncertainties. The methodology uses bond graph language (for system modelling, structural analysis and inverse model generation), and fuzzy logic tools (for uncertainties representation and propagation).

A mechatronic system is classically represented by its transfer function or its state equations. In order to better adapt the modelling to physical phenomena and causalities, we decide, in this paper, to use bond graph language [1] to represent our mechatronic model. It is a multi-discipline language, which facilitates the representation of multi-domain systems. Bond graph is popularly used in engineering applications [2], [3], especially in the case of multi-domain physical system [4], [5]. Its mathematical foundation is established in [6].

The bond graph framework provides users with model inversion algorithm [7], [8]. This approach reduces the number of simulating iterations, as well as the calculus time. It has already been applied in [9], [10], [11]. Based on the concept of bicausality [12], the bond graph inverse model serves for sizing purposes while keeping the same structure as the direct model. The invertibility of a bond graph model is easily checked with existing procedures based on power lines [8] and causal paths [11].

Epistemic uncertainties represent the incompleteness of knowledge about a property or a value, due to insufficiently accurate data. Epistemic uncertainty usually appears along with the vagueness in linguistic explanation: for example " $x$ is about 31". In the simplest form, epistemic uncertainty is often quantified with interval arithmetic [13], [14]. However, it is proven to be expensive in computation time and the propagation result is often over-estimated. Among alternative epistemic uncertainty quantification methods (e.g. imprecise probability, Dempster-shafer evidence theory [15], [16]), we focus here on fuzzy logic [17].

We shall use fuzzy sets to represent outputs uncertainties specifications. In particular, fuzzy operations handle multiple uncertainties criteria on one output. Moreover, uncertainties propagation through inverse model is processed using extension principle.

The paper is organized as follows. The tolerance synthesis problem is formulated in section II. Section III presents the methodology of tolerance synthesis. Section IV illustrates this methodology on a DC motor where both cases of output mono- 
uncertainty and output multi-uncertainties are considered.

\section{Formulation OF THE PROBLEM}

Our goal is to determine the tolerances of design parameters knowing a fuzzy representation of output uncertainties.

System's specifications contain the deterministic behaviour that characterizes the (ideal) scenario to be followed. The associated set of desired output trajectories is then considered to be subject to epistemic uncertainties. Output uncertainties are translated into a family of trajectories living in the neighbourhood of the desired ones, defining the fuzzy behaviour. The model gives us the relation between the fuzzy behaviour and the design parameters, which sets a base of knowledge for tolerance synthesis.

Let us consider a given mechatronic system. We shall use fuzzy logic to quantify the output epistemic uncertainties included in the specifications. Fuzzy logic considers the set in which the membership is gradual and not necessarily Boolean.

\section{Definition 1: [18]}

- If $X$ is a collection of objects denoted generally by $x$, then a fuzzy set $\tilde{A}$ in $X$ is a set of ordered pairs:

$$
\tilde{A}=\left\{\left(x, \mu_{\tilde{A}}(x)\right) \mid x \in X\right\}
$$

$\mu_{\tilde{A}}(x)$ is called the membership function or grade of membership of $x$ in $\tilde{A}$ that maps $X$ to the membership space (such as $[0,1]$ or $\mathbb{R}_{+}$).

- The support of a fuzzy set $\tilde{A}, \operatorname{supp}(\tilde{A})$, is the crisp set of all $x \in X$ such that $\mu_{\tilde{A}}(x)>0$.

- The (crisp) set of elements that belong to the fuzzy set $\tilde{A}$ at least to the degree $\alpha$ is called the $\alpha$-level set or $\alpha$-cut.

$$
A_{\alpha}=\left\{x \in X / \mu_{\tilde{A}}\{x\} \geq \alpha\right\}
$$

- A fuzzy number $\tilde{n}$ is a fuzzy set defined by a normalized, convex, upper semi-continuous membership function with bounded support and unique modal value. It represents an imprecisely known real number $x_{0}$. An example of triangular fuzzy number is depicted in figure 1 below.

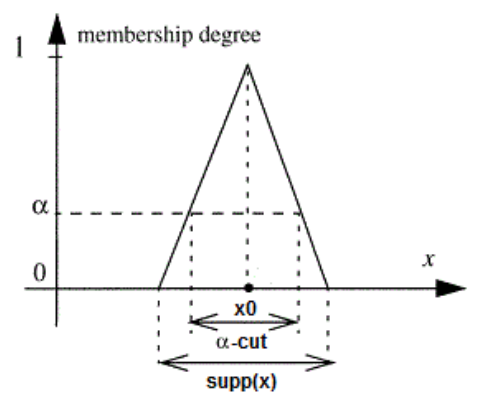

Fig. 1. Membership function of a triangular fuzzy number

Once the fuzzy output behaviour is quantified, the aim is to link it with the design parameters. The inverse model gives us this link with an explicit relation between fuzzy outputs and design parameters. The fuzzy output behaviour is then propagated through the inverse model using ZADEH extension principle.

Theorem 1: Zadeh extension principle [18] Let $X$ be a Cartesian product of universes $X=X_{1} \times \cdots \times X_{r}$ and $\tilde{A}_{1}, \tilde{A}_{2}, \ldots, \tilde{A}_{r}$ be $r$ fuzzy sets in $X_{1}, \ldots, X_{r}$ respectively. $f$ is a mapping from $X$ to a universe $Y, y=f\left(x_{1}, \ldots, x_{r}\right)$. Then the extension principle allows us to define a fuzzy set $\tilde{B}$ in $Y$ by:

$$
\tilde{B}=\left\{\left(y, \mu_{\tilde{B}}(y)\right) \mid y=f\left(x_{1}, \ldots, x_{r}\right), \quad\left(x_{1}, \ldots, x_{r}\right) \in X\right\}
$$

where $\mu_{\tilde{B}}(y)=$

$\left\{\begin{array}{l}\sup _{\left(x_{1}, \ldots, x_{r}\right) \in f^{-1}(y)}\left\{\mu_{\tilde{A_{1}}}\left(x_{1}\right), \ldots, \mu_{\tilde{A_{r}}}\left(x_{r}\right)\right\}, \text { if } f^{-1}(y) \neq \emptyset \\ 0 \quad \text { otherwise }\end{array}\right.$ where $f^{-1}$ is the inverse of $f$.

Propagating fuzzy output behaviour yields the membership functions of the design parameters. Then, from the supports of the parameter membership functions together with the required satisfaction levels, we are able to determine the tolerances of design parameters with respect to the specifications.

\section{Methodology of tOleRAnCE SYNTHESIS IN THE PRESENCE OF UNCERTAINTY}

We propose here the procedure to process epistemic uncertainties in the problem of parametric tolerance synthesis.

A Modelling: Construct the bond graph of the system, model the epistemic uncertainties included in the output specifications with fuzzy logic, determine the set of design parameters and outputs.

B Adequacy: Check the adequacy between the model structure and the input/output specifications [19].

C Inversion: Test the structural invertiblity [8] and construct the inverse bond graph model [20], [19].

D Propagation: Compute design parameter uncertainties by propagating output uncertainties through the inverse model.

E Tolerance synthesis : Synthesize the tolerance of the design parameters from their computed uncertainties.

F Validation : Simulate direct model output behaviours with the synthesized tolerances.

\section{A. System and epistemic uncertainties modelling}

We construct the bond graph model based on the physical phenomena of the (deterministic) mechatronic system. Since we concentrate on the problem of a parameter tolerance synthesis, the structure of the model is assumed to be known and fixed (for instance, there is no black-box, models commutation, discontinuity). From the specifications, we classify the parameters into two sets: the set of known parameters, and the set of design parameters (those of interest for tolerance synthesis). We model the output uncertainties with fuzzy logic according to the specifications. 


\section{B. Adequacy verification}

It is necessary to verify that the desired output behaviours are possible with the model structure and the specified inputs. For many reasons, some specifications may not be compatible with the model dynamics, with the model workspace, etc... A verification of adequacy between the model's structure and the specifications is therefore essential. In practice, adequacy is checked following [19].

\section{Inversion}

Our approach is based on inverse model in order to get an explicit relation between the fuzzy outputs and the design parameters. The direct model outputs will be the inputs of the inverse model, and the design parameters will be the outputs of the inverse model. We shall check that the bond graph model is invertible from the outputs to the set of design parameters. The bond graph framework provides users with invertibility criteria [8]. The inverse model with minimum order is obtained from the bicausal bond graph with the procedure detailed in [19].

\section{Propagation}

The fuzzy output behaviour propagation is processed using ZADEH extension principle [18] applied to the outputs/parameters relation obtained via the inverse model. As a result, we obtain the membership functions of the design parameters according to the fuzzy output behaviours.

\section{E. Tolerance synthesis}

The common support of the membership functions of a parameter contains all the values that satisfy all the fuzzy output behaviours. The associated membership function is the base of knowledge for tolerance synthesis. Hence, depending on the specified satisfaction levels, an $\alpha$-cut gives the corresponding tolerance of the parameter. The knowledge of the parameter membership functions actually gives the system designer flexibility on the tolerance synthesis since $\alpha$-cuts can be actualized with respect to the target satisfaction levels.

\section{F. Validation}

From the obtained tolerances, we generate sample values of the design parameters. We then re-inject this set of values into the direct bond graph model. Simulation results allow to compare the output behaviours with the required specifications.

\section{EXAMPLE}

Consider a DC motor rotating a load (Figure 2). As an illustration of our methodology, we process the tolerance synthesis of the internal resistance of the DC motor. We shall study a simple output mono-uncertainty case and an output multi-uncertainties case.

Modelling assumptions: The electrical part contains a voltage source $u$, an internal resistance $R$ and an inductance $L$. The (ideal) electromechanical coupling is characterized by a torque constant $k_{c}$. The mechanical part takes into account the motor axis inertia $J_{m}$ and the load inertia $J_{c}$, a reduction gear ratio $1 / N$ and the viscous friction coefficient $b_{c}$ on the load axis.

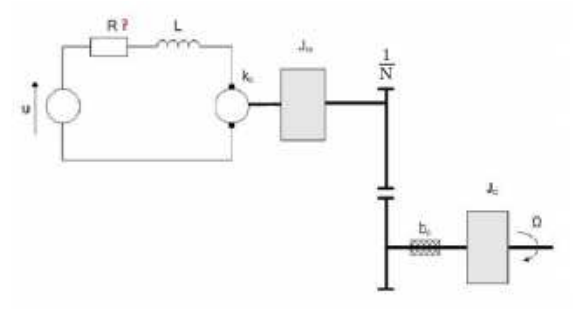

Fig. 2. Scheme of a DC motor rotating a load.

\section{Data specifications.}

$L \quad$ Motor self inductance $\quad 0.001[\mathrm{H}]$

$k_{c} \quad$ Electromechanical coupling $\quad 0.031[\mathrm{~N} . \mathrm{m} / \mathrm{A}]$

$J_{m} \quad$ Motor axis inertia

$N \quad$ Gear ratio

$J_{c} \quad$ Load inertia

$b_{c} \quad$ Viscous friction coefficient $0.0001\left[\mathrm{~N} . \mathrm{m} / \mathrm{rad}_{\mathrm{s}} \mathrm{s}^{-1}\right]$

$u$ Input voltage 20 [V]

Performance specifications. The output angular velocity $\Omega$ is desired to follow a second order step response with an amplitude $K=32 \mathrm{rad} / \mathrm{s}$, a damping ratio $\xi=24$ and an undamped frequency $\omega_{n}=650 \mathrm{~s}^{-1}$.

Uncertainty specifications. The stationary output velocity can vary in the interval $\delta K= \pm 1 \mathrm{rad} / \mathrm{s}$. However, for system security, it must stay at the worst within $70 \%$ of the ideal performance. For the other two parameters, one specifies $\delta \xi= \pm 3$ and $\delta \omega_{n}= \pm 30 \mathrm{~s}^{-1}$. These variations form an envelop that the output trajectory is expected to lie within.

We shall first study the mono-uncertainty case where only epistemic uncertainty on the amplitude $K$ is taken into account. Secondly, we study the multi-uncertainty case where $K, \xi, \omega_{n}$ are considered.

\section{A. The output mono-uncertainty case}

Modelling. The bond graph model of the system is given in figure 3. The internal motor resistance $R$ is the design

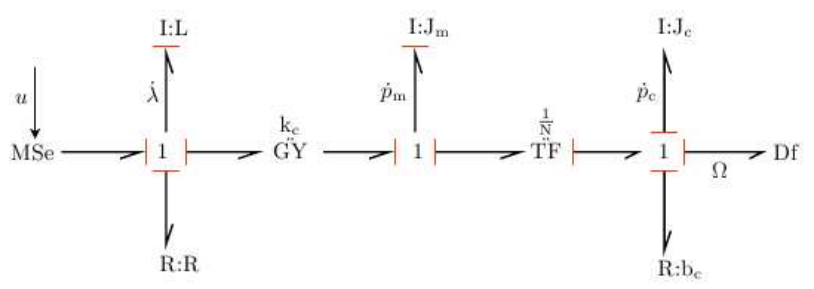

Fig. 3. Causal bond graph representation of a DC motor rotating a charge

parameter and the output is the angular velocity $\Omega$.

The specified uncertain amplitude $K=32 \pm 1[\mathrm{rad} / \mathrm{s}]$ is quantified by a symmetric triangular fuzzy number $\mu_{K}$

$$
\mu_{K}=\mathcal{T}(32,1) \text {. }
$$

Adequacy verification. Following the procedure in [19], we can verify the adequacy of the model with respect to the output 
specifications. Note that in this example, adequacy is trivial since the model leads to second order transfer function and the output specifications require a second order step response.

Inverse model. The structural analysis of the bond graph model shows that it is invertible from the output $\Omega$ to the design parameter $R$. The bicausal bond graph model is given in figure 4.

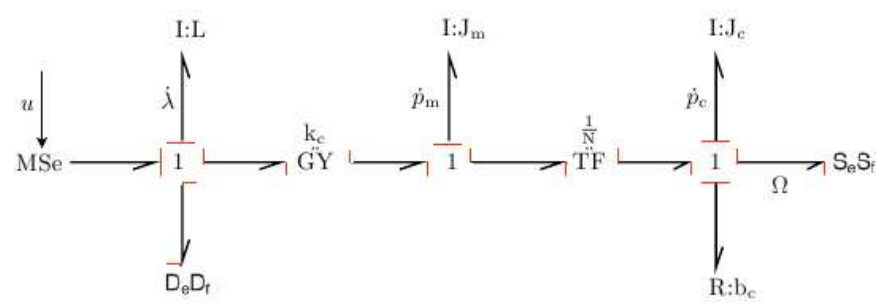

Fig. 4. Bicausal bond graph representation of inverse model

The minimum order inverse model, obtained from [19] applied to 4 , is given by

$$
R=\frac{u-L\left[\frac{1}{k_{c}}\left(\frac{J_{m}}{n}+n J_{c}\right) \ddot{\Omega}-\frac{1}{k_{c}}\left(\frac{b_{m}}{n}+n b_{c}\right) \dot{\Omega}\right]-\frac{k_{c}}{n} \Omega}{\frac{1}{k_{c}}\left(\frac{J_{m}}{n}+n J_{c}\right) \dot{\Omega}+\frac{1}{k_{c}}\left(\frac{b_{m}}{n}+n b_{c}\right) \Omega}
$$$$
=g(u, \Omega, \dot{\Omega}, \ddot{\Omega})
$$$$
=h\left(u, K, \xi, \omega_{n}\right) \text {. }
$$

Propagation. Uncertainty on the amplitude $K$ is propagated to the internal resistance $R$ through the inverse model $h$ given by (1). Note that $\Omega, \dot{\Omega}, \ddot{\Omega}$ are functions of $K$, hence their membership functions are deduced from $\mu_{K}$ the membership function of $K$. The propagation is then processed by applying the extension principle on the relation (1). This results in the membership function $\mu_{R}$ which represents the uncertainty on the design parameter $R$ evolving in time (Figure 5).

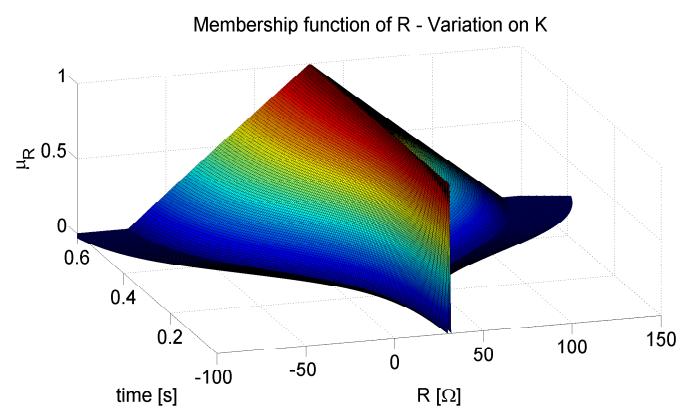

Fig. 5. Membership function of $\mathrm{R}$ evolving in time

Tolerance synthesis. The values of $R$ that partly satisfy the specifications are found in the support of $\mu_{R}$. According to the specifications (at least $70 \%$ of the desired performance), we restrict the support to the $\alpha$-cut of $\mu_{R}$ at 0.7 as shown in figure 6 . The tolerance of $R$ is therefore the smallest interval of the $0.7 \alpha$-cut.
However, since the amplitude uncertainty mainly acts on the steady-state values of the response and little on the transient response, we shall determine the tolerance of $R$ from the $\alpha$-cut at an ad hoc time. In this example, we have chosen 0.1 second. We then conclude that $R$ belongs to the interval [30.06, 31.96]. The output trajectory is therefore expected to stay inside our envelop from 0.1 second.

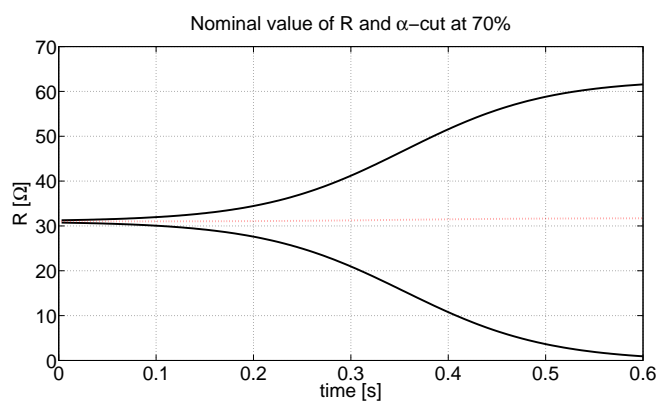

Fig. 6. $\quad \alpha$-cut of $\mu_{R}$ at 0.7 .

Validation. We generate random samples of $R$ within the tolerance interval $[30.06,31.96]$ and inject these values into the direct model. We notice that the corresponding steady states (i.e. the stationary angular velocities) lie in the demanded interval $32 \pm 0.3$ (Figure 7).

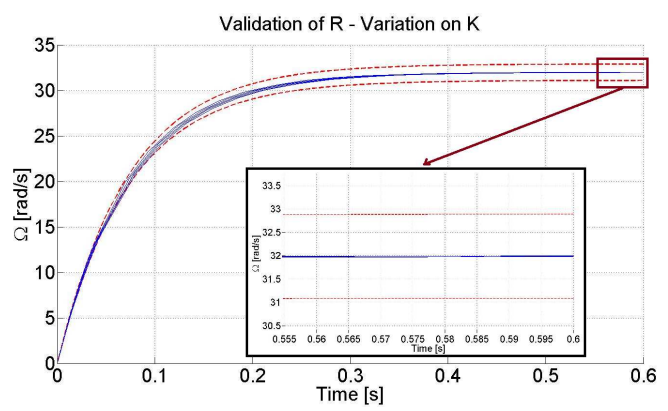

Fig. 7. Simulated trajectories - Variation on K

Moreover, we also notice that some trajectories exceeded the specified envelop during transient response (Figure 8). This was expected since the tolerance synthesis fulfils requirements from 0.1 second. After that time, we guarantee that all trajectories return into the envelop.

We therefore validate the tolerance synthesis on $R$.

\section{B. The output multi-uncertainty case}

Modelling. The modelling assumptions are identical to the previous case. We thus end with the same bond graph model given in figure 3 . The only difference resides in the fact that we now add the damping ratio $\xi$ and the undamped frequency $\omega_{n}$ uncertainties in order to take into account transient response uncertainties. The epistemic output specifications then translates into symmetric fuzzy numbers given by

$$
\mu_{K}=\mathcal{T}(32,0.3), \quad \mu_{\xi}=\mathcal{T}(24,3), \quad \mu_{\omega_{n}}=\mathcal{T}(650,30)
$$




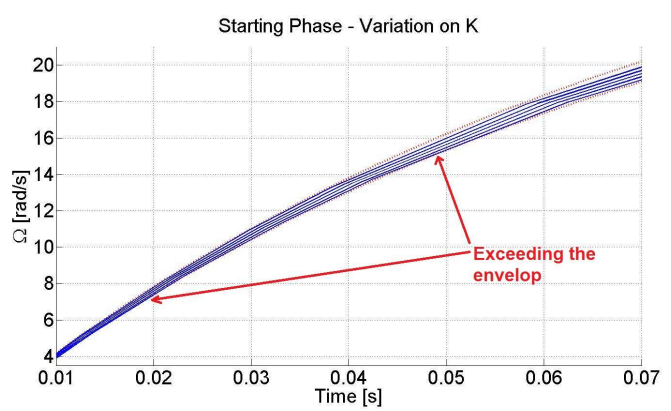

Fig. 8. Zoom into starting phase - Variation on $\mathrm{K}$

Adequacy verification. As before, adequacy is checked following the procedure in [19].

Propagation. Uncertainties of the vector $\left(K, \xi, \omega_{n}\right)$ are propagated to the design parameter $R$ through the inverse model $h$ given in (1). As before, the contribution of the angular velocity and its derivative are replaced by functions of $K, \xi$ and $\omega_{n}$, and so do their corresponding membership functions. The propagation is then processed by applying the extension principle to the equation 1 . We obtain the membership function of $R$ (Figure 9)

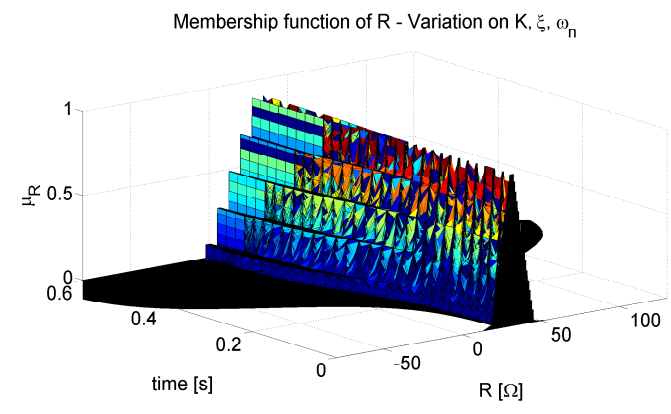

Fig. 9. Membership function $\mu_{R}$ of $\mathrm{R}$ evolving in time

Tolerance synthesis. The time evolution of the support of $R$ is given in figure 10. We shall now synthesize the tolerance as the intersection of all the supports of the memberships function of $R$. That is to say, we consider the smallest interval in time. We conclude that $R$ belongs to $[25.67,36.88]$.

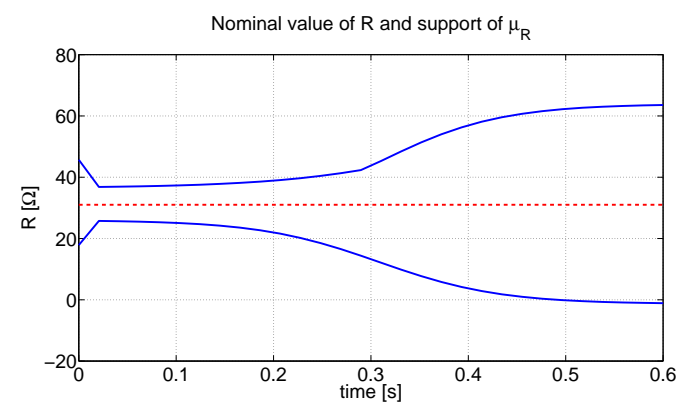

Fig. 10. Nominal value of $R$ and support of $\mu_{R}$

Validation. We generate random samples of $R$ within the interval $[25.67,36.88]$ and inject these values into the direct model. The simulation results are given in figure 11. In the present case, all the trajectories stay in the specified envelop.

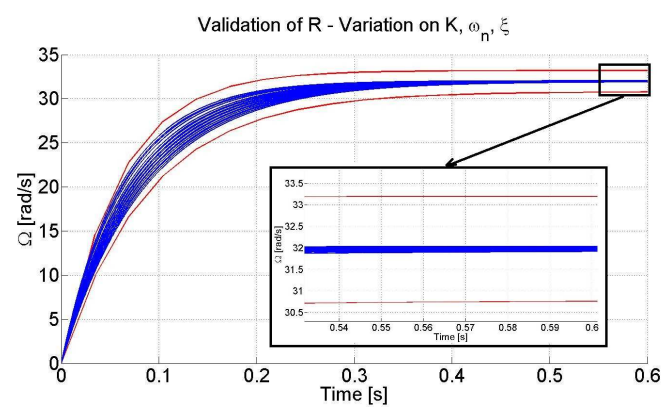

Fig. 11. Simulated trajectories - Variation on $\mathrm{K}, \omega_{n}, \xi$

Moreover, on the contrary to the previous case, the transient behaviour is fully fulfilled as shown in figure 12. This shows the necessity to address tolerance synthesis the with respect to the whole set of specifications. In fact, it is easy to see that an uncertainty has an effect on all the characteristics of the model outputs, not only their stationary values, but also their transient behaviour. As a consequence, it is reasonable to uncertainty specifications on all of these output parameters.

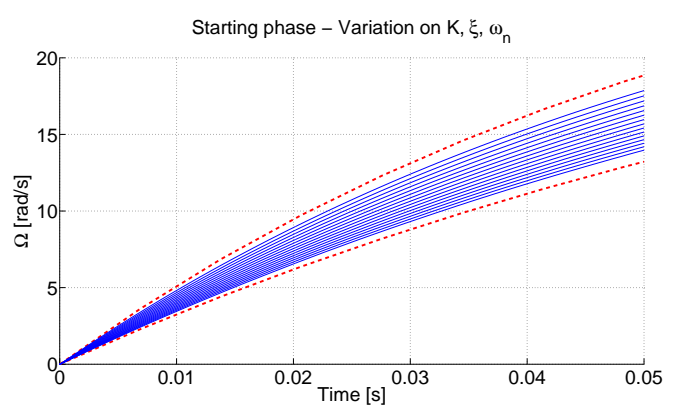

Fig. 12. Zoom into starting phase - Variation on $\mathrm{K}, \omega_{n}, \xi$

We furthermore validate the tolerance synthesis on $R$.

\section{CONCLUSION AND OUTLOOK}

In this paper, we presented the problem of mechatronic system design in the presence of epistemic uncertainty, and particularly the problem of parametric tolerance synthesis.

We chose the bond graph language as the modelling tool, because of its multi-disciplinary and acausality. The adopted approach for parametric sizing is the methodology of inversion. That methodology proves its interest over the direct approach in term of calculation cost. However, it requires the structure of model to be invertible.

Epistemic uncertainty is modelled by fuzzy numbers and propagated using the extension principle of ZADEH. This principle was chosen, for the sake of generality of the methodology: the extension principle works for fuzzy sets and, as a result, is still applicable when the uncertainty becomes more 
complex and can no longer be represented by a single fuzzy number.

The proposed methodology provides designers with the membership function of the parameter under consideration, evolving in time, which offers a great flexibility on tolerance synthesis. It gives also the possibility to treat multiple specifications at the same time, regarding that we can use fuzzy operations to combine corresponding membership functions.

The methodology was illustrated via a simple example of sizing issue on a DC motor. The synthesized tolerance satisfied the initial specifications and therefore, validated the methodology.

As we mentioned above, the proposed procedure is used to deal with epistemic uncertainty in the design process. However, there are scenarios where aleatory and epistemic uncertainties coexist in the specifications. We can classify the uncertainty in the specifications into two types: uncertainty on technical specifications and uncertainty on statistical specifications. The technical specifications are normally given from customer's demand or expert's experience. They contain relative ignorance upon information. The statistical specifications are often given on the manufacturing process. However, these two types of uncertainty sometimes can not be treated separately. A common situation is where one may know the form of the probability distribution for an uncertain variable, but not be sure about the parameter giving the distribution (inexact expectation and variation of a distribution, for example). Another situation is where the two types of uncertainty cross over in a complex specification. For example: "60\% of the designed components $x$ satisfies more than $90 \%$ of technical demand, and has a cheap fabrication cost." . In this case, " satisfies technical demand" and "has cheap fabrication cost" are two epistemic uncertainties on the specification and "60 $\%$ of the designed components" is statistical uncertainty. This type of uncertainty can not be handled by either fuzzy logic or probabilistic tools. A combined representation is necessary.

In order to propagate both aleatory and epistemic uncertainties through model, we can propagate them separately as the proposed method in this paper and in [19]. Another way is the second-order probability method [21]. However, it is expensive since two sampling loops are required.

The modelling and propagation of both aleatory uncertainty and epistemic uncertainty are complex and expensive in calculation. However, it gives us a more complete and general understanding on system, as well as the possibility to deal with complex specifications in design progress.

Another outlook for the methodology is the generalization to multi-uncertainties case. In fact, the methodology contains already the possibility of handling the multi-uncertainties problem, considering that we can regroup all the variables into a vector and treating them as one. However, when the variables are no longer independent, the result's membership function may end up with a very large support, due to the effect of overestimation. In [22], a transformation method to reduce the error of over-estimation is proposed. However, the implementation of the transformation method to our propagation process should only be done within consideration about the calculation cost.

\section{REFERENCES}

[1] H. Paynter, Analysis and Design of Engineering Systems, ISBN.0262160048 ed. Cambridge, USA: The M.I.T Press, 1961.

[2] P. C. Breedveld, "Modeling and simulation of dynamic systems using bond graphs," In Control Systems, Robotics and Automation, from Encyclopedia of Life Support Systems (EOLSS), Developed under the Auspices of the UNESCO. EOLSS Publishers, p. 1-36, 2008.

[3] D. Karnopp, D. Margolis, and R. Rosenberg, System Dynamics. Modeling and Simulation of Mechatronic systems, 4th ed. Hoboken, New Jersey: John Wiley \& sons, Inc, 2012.

[4] P. Breedveld, "Port-based modelling of multidomain physical systems in terms of bond graphs," Simulation Techniques for Applied Dynamics, p. 141-190, 2009.

[5] D. Karnopp, "Bond graph models for electrochemical energy storage : electrical, chemical and thermal effects," Journal of the Franklin Institute, vol. 327, no. 6, pp. 983-992, 1990.

[6] S. Birkett and P. Roe, "The mathematical foundations of bond graphs-I. algebraic theory," Journal of the Franklin Institute, vol. 326, no. 3, pp. 329-350, 1989.

[7] S. Scavarda, M. Amara, and E. Richard, "Determination of the output power in terms of output variables using bond graph," IMACS-IFAC Symposium MCTS 91 Modelling and Control of Technological systems, p. 15,1991

[8] R. F. Ngwompo, S. Scavarda, and D. Thomasset, "Inversion of linear time-invariant SISO systems modelled by bond graph," Journal of the Franklin Institute, vol. 333, no. 2, pp. 157-174, Mar. 1996.

[9] O. Mechin, W. Marquis-Favre, S. Scavarda, and P. Ferbach, "A dynamic sizing of an electronic power steering system," ICBGM'03 International Conference on Bond Graph Modeling, pp. 137-147, 2003.

[10] A. Jardin, W. Marquis-Favre, D. Thomasset, F. Guillemard, and F. Lorenz, "Study of a sizing methodology and a modelica code generator for the bond graph tool MS1," in Proceedings of the 6th International Modelica Conference, Bielefeld, Allemagne, Mar. 2008, pp. $125-134$.

[11] A. Jardin, W. Marquis-Favre, and D. Thomasset, "Bond graph sizing of mechatronic systems: Coupling of inverse modelling with dynamic optimization," in Proceedings MATHMOD 09 Vienna - Full Papers CD Volume, F. B. I. Troch, Ed., Vienne, Autriche, Feb. 2009, pp. 1929-1938, ISBN 978-3-901608-35-3.

[12] P. Gawthrop, "Bicausal bond graphs," SOCIETY FOR COMPUTER SIMULATION, vol. 27, pp. 83-88, 1995.

[13] J. C. Helton, J. D. Johnson, W. L. Oberkampf, and C. J. Sallaberry, "Representation of analysis results involving aleatory and epistemic uncertainty," International Journal of General Systems, vol. 39, no. 6, p. 605-646, 2010

[14] J. Helton, J. Johnson, and W. Oberkampf, "An exploration of alternative approaches to the representation of uncertainty in model predictions," Reliability Engineering \& System Safety, vol. 85, no. 1-3, pp. 39-71, Jul. 2004.

[15] G. Shafer, A mathematical theory of evidence. Princeton university press Princeton, 1976, vol. 1.

[16] W. L. Oberkampf and J. C. Helton, "Investigation of evidence theory for engineering applications," in Non-Deterministic Approaches Forum, 43rd AIAA/ASME/ASCE/AHS/ASC Structures, Structural Dynamics, and Materials Conference, 2002.

[17] L. Zadeh, "Fuzzy sets," Information and Control, vol. 8, no. 3, pp. 338353, Jun. 1965

[18] J. Hans, Fuzzy Set Theory-and Its Applications, 2001.

[19] M. E. Feki, "Analyse et synthèse de tolérance pour la conception et le dimensionnement des systèmes mécatroniques," Ph.D. dissertation, Ecole Centrale de Lyon, Jul. 2011.

[20] A. Jardin, "Contribution à une méthodologie de dimensionnement des systèmes mécatroniques : analyse structurelle et couplage à l'optimisation dynamique," Ph.D. dissertation, Jan. 2010.

[21] S. Laura P, P. Thomas L, and M. Randall L, "Epistemic uncertainty quantification tutorial," 2009.

[22] M. Hanss, "The transformation method for the simulation and analysis of systems with uncertain parameters," Fuzzy Sets and Systems, vol. 130, no. 3, pp. 277-289, Sep. 2002. 